\title{
Study of Semiotic Aspect in Youth Communication Based on Neurotechnologies
}

\author{
Julia Yeremenko ${ }^{1},{ }^{*}$ Oksana Ulanovskaya ${ }^{1}$, Elena Remesnik ${ }^{1}$ \\ ${ }^{1}$ V.I. Vernadsky Crimean Federal University, Russia \\ *Email:jul_eremenko@mail.ru
}

\begin{abstract}
This paper explores the features of youth perception of the message semiotic aspect and its impact on the communication process effectiveness through the use of neurotechnologies. A neurophysiological study using a stationary IT tracker made it possible to obtain new objective data on the level of visual attention, interest, emotional involvement, and memorability of a communicative message containing symbols significant for the target audience. The results of the research have shown that at the conscious level, test persons do not feel differences in the assessment of communicative messages with the presence/absence of symbols significant for them. However, the study of unconscious neurophysiological reactions has indicated several meaningful distinctions in perceiving the stimulus material. Thus, the percentage of memorability of a communicative message (image together with text) with the availability of visual symbols is higher among recipients having an emotional response to them: for group N1 it is $63.33 \%$, for group $\mathrm{N} 2$ $46.43 \%$. The study has also revealed the differences in an average duration of fixations on a picture-symbol at the 0.1 level of significance, which indicates the increase of visual attention and its influence on memorability.
\end{abstract}

Keywords: Social communication, Intergenerational communication, Symbolic interactionism, Symbol, Neurotechnology, Oculographic research.

\section{INTRODUCTION}

Studying various aspects of forming effective communication among young people is gaining more and more scientific and, above all, social significance. They are related directly to personality socialization, the transfer, and formation of values in society, the accumulation and consolidation of social experience, as well as building relationships between people. However, our research, carried out in the youth (in particular, student) environment, records an increase in the volume and intensity of information flows, and the complexity of intergenerational communication. As a result, it becomes increasingly difficult to "reach out" to young people to convey the social meaning of messages. Thus, it becomes relevant to search the "triggers" for launching an effective communication process capable of making the message "visible" in a redundant information environment, understandable to a specific target audience, and able to trigger the necessary social reaction.
The gaps in intergenerational communications are getting more and more frequently identified by traditional sociology, which is facilitated to a great extent by outwardly attractive, but not always socially justified experiments in education, as well as by the advertised "online learning" system. In combination, these two trends are focused on breaking intergenerational links in the key to transferring social experience in the form of unpretentious "messages - axioms". As a result, there is a significant shift in the value orientations of youth.

However, in order not just to fix these gaps, but to consider their causes and internal mechanisms, a systematic, comprehensive approach is needed. A social and scientific explanation of the process taking place in society has revealed its limitations if the data of these sciences are used in isolation. Mutual systemic registration of data from these areas of scientific knowledge allows us to seriously enrich and deepen our understanding of social life [1]. Sociological theory must be physically and computationally realistic: if social 
phenomena are related to cognitive processes, sociology must be able to describe them adequately [2].

This paper presents an example where the synthesis of sociological and neurophysiological explanations of how young people perceive cultural symbols and how they influence the effectiveness of communication.

\section{SYMBOLIC SOCIAL COMMUNICATION}

In our study, we will address the interactive (nonclassical) paradigm, in the context of which the central themes are: interpersonal communication, forms of intercultural communication, the creation of a common system of symbols and signs, as well as the formation of identity in another culture. In sociology, this paradigm is mainly implemented in the theory of symbolic interactivity, as well as the traditions of subsequent theories that follow it.

The central element of symbolic interactionism is the concept introduced by the American philosopher J. Mead, "a sign symbol", i.e. a subject, a gesture, which is consciously used in the course of social interaction to transfer meaning from one person to another and which has the same meaning for both the transmitter and the perceiver [3].

The key problem with the symbols using in communication is the very nature of the symbol, the essence of which is not static, but rather a dynamic process that depends on the interpretation of the relationship between the sign and the object. The interpretation process is influenced by many factors: context, participants in the communication process, language, and others, which leads to a variety of meanings and possible misunderstandings that make social communication impossible. It should be noted that the main condition of social communication is the correct transmission of a message (in any form) and the correct interpretation of its meaning by the recipient.

Thus, the symbols using in communication require careful study, since, on the one hand, they can provide additional meaning and significance to the message, on the other may cause the effect of non-congruence, additional cognitive load, and lead to misunderstanding and irritation. The application of sociological and neurophysiological approaches to the study of symbol perception features in a communicative message can provide new knowledge about modeling an effective communication process in young people.

\section{NEUROPHYSIOLOGICAL METRICS TO ASSESS THE PERCEPTION OF VISUAL STIMULI}

Obtaining objective information about the perception of visual stimuli is associated with oculographic studies.
They allow a comprehensive study of visual attention in information processing, which makes it possible to identify preferences and dependencies on various cognitive processes, such as attention and interest.

Visual attention is considered as a set of cognitive operations aimed at selecting the relevant, and filtering out unnecessary information from oversaturated visual scenes [4]. Several scientific papers show the dependence of visual attention on interest and memorability [5-8].

The largest theoretical base of knowledge on the application of various visual images in communication has been developed in the field of marketing, namely advertising communications, where the influence of celebrities, characters of virtual games, elements of nature or cultural symbols on the economic behavior of consumers, brand memorability and brand positioning has been studied [9-12].

It can be assumed that the symbols identified in the course of sociological research, significant for a particular youth audience, have a high emotional load which could activate the subconscious and cause strong memories and interest, which will have a positive impact on the communication process. In our case, the metrics used to evaluate the effectiveness of communication were the rating indicators of message evaluation, obtained using the Likert scale, as well as neurophysiological indicators of visual attention, interest, and memorability, obtained using a stationary eye tracker.

\section{RESEARCH METHODOLOGY}

This paper is based on the analysis of the results of sociological and neurophysiological research conducted by the authors in March-May 2019 at the Laboratory of Neuromarketing and Behavioral Economics of V.I. Vernadsky Crimean Federal University.

The research of semiotic aspect perception peculiarities in youth social communication consisted of two consecutive stages: sociological research of youth subculture semiotics (selection stage) and neurophysiological research aimed at studying unconscious reactions of a person to stimuli.

\subsection{Stage. Sociological research}

To achieve the goal of the research, the sociological study had to answer questions related to the definition of common parameters uniting respondents and identification of a symbolic coding system within the selected parameters, which are necessary for the development of incentive material for the second stage of the research. The character tendency of modern youth is the synthesis of sociosemiotic characteristics on musical, philosophical, aesthetic, and other grounds. The most common parameter, uniting young people from the chosen set, was the "film genre", namely a fantastic 
movie. The most frequently mentioned fantastic movies were "Harry Potter" and "Game of Thrones". The attributes associated with the corresponding movies were: a magic wand, glasses, a wizard's hat, a Gryffindor's scarf, an iron throne, a dragon, a castle, a three-eyed raven, a tree of life, a dire wolf.

Thus, the results of a sociological survey and focus group aimed at clarifying the significant attributes of the movie (objects, images, signs), made it possible to prepare an incentive material for the development of eyetracking research and select 2 experimental groups, where N1 is a group for which the images from the movie "Harry Potter" are symbolic, N2 is a group that experiences a neutral attitude to the presented symbols, or does not know them.

\subsection{Stage. Neurophysiological research}

The main study objective achievement is associated with neurophysiological research aimed at studying the unconscious reactions of a person in the process of perceiving a communicative message containing/not containing symbols significant for him/her. The purpose of the experiment is to identify the peculiarities of perception of a communicative message with the presence/absence of symbols significant for the recipients, based on oculographic data.

Obtaining objective quantitative data as a result of eye-tracking research will measure the level of attention, interest, memorability, emotional involvement of the recipient in the process of social communication, which serve as indicators to assess the effectiveness of the communicative process. The following hypotheses have been formulated as part of the second stage of the study:

H1. The presence of a significant visual symbol in a communicative message influences the recipient's ranking.

H2. Indicators of memorability of a communicative message with the presence of visual symbols will be higher in recipients who have an emotional response to these symbols.

H3. The number and average duration of fixations on a symbol will be higher for recipients who have an emotional response to them.

H4. The memorability of the visual image in a communication message correlates with the memorability of the text (verbal part of the message).

\subsubsection{Participants}

The participants were divided into two experimental groups. N1 - a group with a positive emotional response to the presented symbols in a message, $\mathrm{N} 2$ - a group with a neutral attitude to the presented symbols or does not know them.

\subsubsection{Experiment design and procedure}

Before the experiment, the participants were explained the task and also conducted a five-point calibration procedure. All participants have signed an agreement to process their personal data. The participants were shown a slide for $8000 \mathrm{~ms}$, then the Likert scale was presented, where the participants had to perform its evaluation with "like / dislike". The experiment consisted of 10 options of stimulus material. Before each new stimulus was presented, an additional calibration procedure was performed. After the experiment, a survey was conducted to identify the most memorable elements of the message (images and text). Data on memorized images and text in messages were recorded for each participant.

\subsubsection{Equipment and stimulus material}

As a rule, modern messages are creolized, that is, they consist of signs of different semiotic systems (verbal, audio, visual). This paper studies the texts with complete creolization, according to the terminology of E.E. Anisimova (Anisimova, 2003), i.e. consisting of verbal and non-verbal elements - images and text.

As a stimulus material, 10 social messages were used, developed in Adobe Photoshop CS3 graphic program, where the image size and extension of 120 pix/inch were corrected. The content of the messages concerned the call to solve various social problems. $50 \%$ of the messages contained the most famous and recognizable symbols from the "Harry Potter" movie.

\subsubsection{Results of Stage II and their discussion}

Ratings (0-4) were analyzed for each experimental group for stimulus materials and between groups. It was found out that there were no statistically significant differences in rating scores between these groups for any stimulus material. No significant differences were also found in the ratings of two types of stimulus materials for each group individually. Thus, no relationship was found between the presence of a significant symbol in the message and its rating in experimental groups N1, N2, which disproves the hypothesis $\mathrm{H} 1$.

At the same time, the analysis of data on memorized visual and verbal information of the stimulus material revealed certain differences. The percentage of communicative message memorability (images together with text) with the presence of visual symbols is higher for recipients who experience an emotional response to them: for group N1 it is $63.33 \%$ and $46.43 \%$ for $\mathrm{N} 2$. The percentage of text memorability does not differ as much between experimental groups as the percentage of image memorability. The percentage of text memorability for group $\mathrm{N} 1$ is $53.33 \%, 52.38 \%$ for group $\mathrm{N} 2 ; 60.00 \%$ and 
$47.62 \%$ for image respectively. These results support hypothesis $\mathrm{H} 2$.

The result of the neurophysiological experiment was several standard parameters of the participants' oculomotor behavior. The data were collected for experimental groups $\mathrm{N} 1$ and N2, refer to table 1 .

Statistic analysis of the visual fixations on slides by groups was carried out, which showed that the sample data do not differ significantly, which can be seen from the descriptive statistics of the index for experimental groups (table 1).

However, a comparison of groups by the average duration of visual fixation on slides reveals differences. Based on the Mann-Whitney non-parametric criterion, we conclude that there is a statistically significant difference between the groups, i.e. the average duration of fixation on slides for experimental groups differs, which can be seen from the descriptive statistics (table 1).

Statistical analysis of the average duration of fixations on the text does not reveal any differences between the experimental groups, while the average duration of fixations on the image is significantly different, which is visually noticeable in Fig. 3. The data obtained allow us to conclude that the use of visual symbols significant for the recipient in the communicative message leads to increased visual attention, while, as it was noted earlier, the memorability of the communicative message with visual symbols significant for the recipient is higher, which supports the hypothesis $\mathrm{H} 3$.
Besides, it was found that the memorability of the visual image in the communicative message leads to the memorability of text, which is indicated by the Pearson correlation factor $(\mathrm{r}=0.89, \mathrm{p}=0.0006)$. The high correlation between these indicators confirms the hypothesis $\mathrm{H} 4$.

\section{CONCLUSIONS}

1. On a conscious level, the participants do not make any distinction in evaluating communicative messages with the presence/absence of meaningful symbols. No relationship was found between the presence of a significant symbol in the message and its rating in the experimental groups $\mathrm{N} 1, \mathrm{~N} 2$, which rejects the hypothesis $\mathrm{H} 1$.

2. The study showed that both experimental groups had approximately the same amount of memorized stimulus material, but the percentage of memorability of a communicative message (images together with text) with the presence of visual symbols was higher for recipients who had an emotional response to them: $63.33 \%$ for group N1 and $46.43 \%$ for group N2. Obtained results confirm the hypothesis $\mathrm{H} 2$.

3. The presence of emotionally significant symbols for recipients leads to increased visual attention to them and affects their memorability. The statistic analysis of the average duration of fixation on the text does not reveal any differences between experimental groups, while the average duration of fixation on the image is significantly different. The results of the study partially confirm the hypothesis $\mathrm{H} 3$.

Table 1. Pairwise comparison of experimental groups N1, N2

\begin{tabular}{|l|c|c|}
\hline \multicolumn{1}{|c|}{ Data set No. 1 (presence of the image-symbol) } & N1 & N2 \\
\hline Gaze: fixations (count) & $30,77,86$ \\
\hline Gaze: fixations (count/ms) & 2,88 & 178,49 \\
\hline Gaze: duration of fixations. Average (ms) & 189,15 & 121,92 \\
\hline Gaze: duration of fixations. Median (ms) & 127,37 & 189,92 \\
\hline Gaze: duration of fixations. Average (ms) (area of interest: image) & 215,92 & 169,951 \\
\hline Gaze: duration of fixations. Average (ms) (area of interest: text) & 174,81 & $\mathrm{~N} 2$ \\
\hline Data set No. 2 (no image-symbol) & $\mathrm{N} 1$ & 32,71 \\
\hline Gaze: fixations (count) & 31,69 & 3,09 \\
\hline Gaze: fixations (count/ms) & 2,97 & 165,11 \\
\hline Gaze: duration of fixations. Average (ms) & 181,10 & 118,71 \\
\hline Gaze: duration of fixations. Median (ms) & 125,90 & 195,89 \\
\hline Gaze: duration of fixations. Average (ms) (area of interest: image) & 221,78 & 155,21 \\
\hline Gaze: duration of fixations. Average (ms) (area of interest: text) & 153,66 & \\
\hline
\end{tabular}


4. There is a high correlation between image and text memorability of 0.89 . If the participants were able to remember the image in a communication message, they were highly likely to remember the text part. The results of the study confirmed the hypothesis H4.

The results of the study showed that using an image symbol in a message can be a trigger for effective communication among young people, which can increase visual attention and memorability.

Although there was no objective to study the influence of symbols significant for the recipient in the communicative message on long-term memory, the authors conducted an express study which showed that one month after participating in the main experiment, the participants of experimental group N1 were able to recall the content of some of the previously presented stimuli, while the participants of group $\mathrm{N} 2$ did not remember anything.

\section{REFERENCES}

[1] A.V. Shkurko, On the way to neurosociology. Sotsiologicheskie issledovanya [Sociological studies] 4 (2011) 13-22.

[2] S. Turner, Social Theory as a Cognitive Neuroscience, European Journal of Social Theory 10(3) (2007) 357-374.

[3] J. Mead, From gesture to symbol, In: American Sociological Thought: Texts. Moscow: MGU, 1994.

[4] S.A. McMains, S. Kastner, Visual Attention. In: Binder M.D., Hirokawa N., Windhorst U. (eds) Encyclopedia of Neuroscience, Springer, Berlin, Heidelberg, 2009.2 DOI: https://doi.org/10.1007/978-3-540-29678-2

[5] C.M. Masciocchi, S. Mihalas, D. Parkhurst, E. Niebur, Everyone knows what is interesting. Salient locations which should be fixated. Journal of Vision Vol. 25. Iss. 9(11) (2009) 1-22.

[6] L. Itti, Models of Bottom-Up and Top-Down Visual Attention, $\mathrm{PhD}$ thesis, California Institute of Technology Pasadena, 2000.

[7] M. Mancas, O. Le Meur, Memorability of natural scenes: the role of attention. Conference: 2013 20th IEEE International Conference on Image Processing (ICIP), $2013 \quad$ DOI: https://doi.org/10.1109/ICIP.2013.6738041

[8] P. Isola, J. Xiao, D., Parikh, A. Torralba, A. Oliva, What Makes a Photograph Memorable? In IEEE Transition on pattern Anal. Mach. Intell Vol. 36. Iss. 36(7) (2014) 1469-1482. DOI: https://doi.org/10.1109/TPAMI.2013.200
[9] A. Schouten, L. Janssen, M. Verspaget, Celebrity vs. Influencer endorsements in advertising: the role of identification, credibility, and Product-Endorser fit. International Journal of Advertising Vol. 39. Iss. 2 (2020) 258-281. DOI: https://doi.org/10.1080/02650487.2019.1634898

[10] N. Saldanha, R. Mulye, K. Rahman, A strategic view of celebrity endorsements through the attachment lens, Journal of Strategic Marketing 28:5 (2020) 434-454. DOI: https://doi.org/10.1080/0965254X.2018.1534877

[11] H.C. Lin, P.F. Bruning, H. Swarna, Using online opinion leaders to promote the hedonic and utilitarian value of products and services. Business Horizons Vol. 61 Iss. 3 (2018) 431-42.

[12] N.J. Evans, J. Phua, J. Lim, H. Jun. Disclosing Instagram influencer advertising: the effects of disclosure language on advertising recognition, attitudes, and behavioral intent. Journal of Interactive Advertising Vol. 17 Iss. 2 (2017) 13849. 